\title{
deformaciones en el hormigoin por fluencia para un estado tensional discontinuo con el tiempo
}

\author{
José Ramón Rosso Ródenas \\ Ingeniero Técnico de Obras Públicas*
}

450-13

\begin{abstract}
sinopsis Recomendaciones del CEB.FIP de 1970 y en la EH-68 no es válido para las fórmulas de deformaciones por fluencia de las actuales instrucciones EH-73 y EP-77, por lo que constituye un grave inconveniente para proyectar una estructura cuando actúan cargas de una manera discontinua con el tiempo.
\end{abstract}

\begin{abstract}
El objeto de este artículo es el de arbitrar un método que nos permita calcular una curva de fluencia para una descarga intermedia que cumpla con el principio de superposición, admitiendo como ciertas la curva de fluencia de carga y la de deformación elástica diferida para descarga total de las EH-73 y EP-77.
\end{abstract}

Tanto en la Instrucción española de hormigón armado (EH-73) como en la de hormigón pretensado (EP-77), que ha seguido manteniendo idéntico criterio, se ha introducido en la fórmula que define la deformación por fluencia un sumando representativo de la deformación elástica diferida. El tener en cuenta este sumando, obtenido experimentalmente descargando una probeta previamente comprimida durante un cierto tiempo, nos acerca más al fenómeno real pero, como más adelante veremos, nos complica bastante los cálculos cuando, como es normal en muchas estructuras pretensadas, se presentan distintos escalones de tensiones espaciados en el tiempo.

Nuestras instrucciones no solamente no hacen comentario alguno sobre la forma de proceder en estos casos sino que pueden inducir a error aun en el simple supuesto de que quiera conocerse la evolución de la fluencia cuando se realiza una única descarga total.

Con independencia de la exactitud de sus resultados la EH-68 y las Recomendaciones del CEB-FIB de 1970 presentaban un proceso de cálculo para la fluencia que, por aplicar la misma fórmula en caso de incremento o disminución de tensiones, hacían posible el principio de superposición simplificándose los cálculos cuando existían variaciones tensionales prolongadas.

El objeto de este artículo es el de arbitrar un método de cálculo tal que, admitiendo como ciertas la curva de fluencia de carga y la de deformación elástica diferida para descarga total de los EH-73 y EP-77, se pueda calcular una curva de fluencia para una descarga intermedia que cumpla con el principio de superposición.

Supongamos que en el instante $t_{1}$ se somete una probeta a una tensión de compresión determinada y que se descarga súbitamente en el instante $t_{2}$. Antes de realizar esta experiencia la probeta se ha sometido a un proceso de carga y descarga rápida con objeto de eliminar futuras deformaciones instantáneas remanentes. La ley de deformaciones con el tiempo será la $A B C D E$ indicada en la fig: 1; $A B$ y $C D$ serán las deformaciones elásticas instantáneas.

* Director División de Estructuras de IBERING, S. A. 
La deformación por fluencia para un tiempo $t_{3}$ será la $E M$, que se obtendrá como diferencia entre $D N$, deformación total diferida en $t_{2}$ y EP, recuperación elástica diferida correspondiente al período $t_{3}-t_{2}$. Siguiendo las fórmulas dadas por las instrucciones españolas de hormigón:

$$
\begin{aligned}
& \mathrm{EM}=\mathrm{DN}-\mathrm{PE} \\
& \mathrm{DN}=\varphi_{t_{2}} \frac{\sigma}{\mathrm{E}_{c}} ; \varphi_{t_{2}}=\varphi_{o}\left(\beta_{t_{2}-t_{1}}\right)+0,4 \beta^{\prime}{ }_{\left(t_{2}-t_{1}\right)} \\
& \mathrm{DN}=\left[\varphi_{o}\left(\beta_{t_{2}}-\beta_{t_{1}}\right)+0,4 \beta_{\left(t_{2}-t_{2}\right)}^{\prime}\right] \frac{\sigma}{\mathrm{E}_{c}}
\end{aligned}
$$

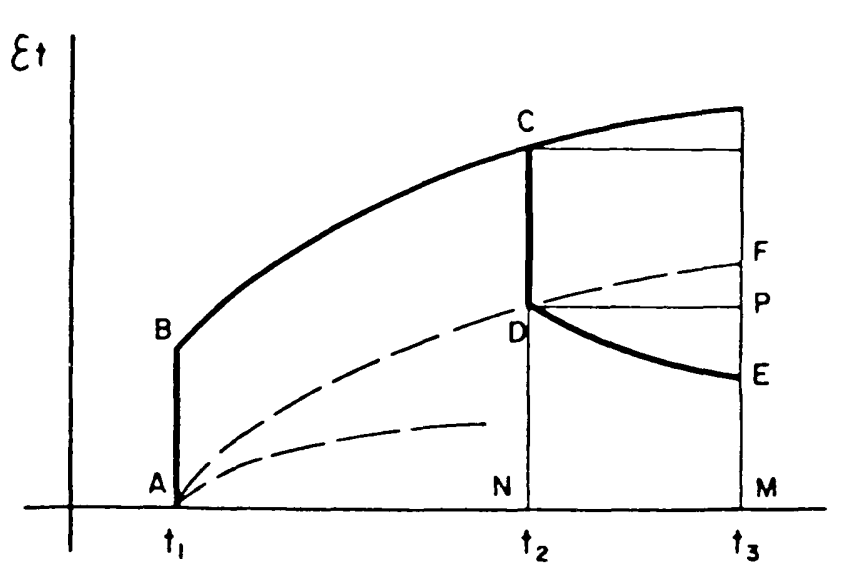

Figura 1

En la fórmula que nos define el coeficiente $\varphi_{\prime \prime}$ el sumando correspondiente a la deformación elástica diferida se obtiene experimentalmente descargando la probeta y viendo cómo evoluciona con el tiempo la recuperación diferida. Por consiguiente, y por definición, EP será la deformación elástica diferida correspondiente al período $t_{3}-t_{2}$.

$P E=0,4 \beta^{\prime} t_{\left.1,-t_{2}\right)} \quad \frac{\sigma}{E_{c}}$

$E M=\left[\varphi_{0}\left(\beta_{t_{2}}-\beta_{t_{1}}\right)+0,4 \beta^{\prime}{ }_{\left(t_{2}-t_{1}\right)}-0,4 \beta^{\prime}{ }_{\left(t_{3}-t_{2}\right)}\right] \frac{\sigma}{E_{c}}$

Supongamos ahora que mantenemos la carga desde $t=t_{1}$, hasta $t=t_{2}$ y que instantáneamente al llegar a $t_{2}$ descargamos y volvemos a cargar la totalidad de la fuerza. Lógicamente cabe admitir que la deformación por fluencia en el instante $t_{3}$ será la misma que si se hubiera mantenido la carga desde $t_{1}$ a $t_{3}$.

Deformación diferida con carga de $\mathrm{t}_{1} \mathrm{at}_{3}$

$\mathrm{MF}=\varphi_{t_{3}} \frac{\sigma}{\mathrm{E}_{c}}=\left[\varphi_{c}\left(\beta_{t_{3}}-\beta_{t_{1}}\right)+0,4 \beta^{\prime}{\left(t_{3}-t_{2}\right)}\right] \frac{\sigma}{\mathrm{E}_{c}}$

La deformación diferida con carga y descarga súbita en $t_{2}$ se descompondrá en dos sumandos, uno correspondiente a la descarga, que es el EM hallado anteriormente, y otro que corresponde a la carga y que equivaldrá a la deformación diferida debida a la fuerza actuando desde $t_{2}$ a $_{3}$. Hallaremos este último valor:

$$
\begin{aligned}
& \varepsilon_{t_{3}}=\varphi_{t_{3}} \quad \frac{\sigma}{\mathrm{E}_{c}}=\left[\varphi_{\mathrm{o}}\left(\beta_{t_{3}}-\beta_{t_{2}}\right)+0,4 \beta^{\prime}{ }_{\left(t_{1}-t_{2}\right)}\right] \quad \frac{\sigma}{\mathrm{E}_{c}} \\
& E M+\varepsilon_{t_{3}}=\left[\varphi_{o}\left(\beta_{t_{2}}-\beta_{t_{1}}\right)+0,4 \beta^{\prime}{ }_{\left(t_{2}-t_{1}\right)}-0,4 \beta_{\left(t_{3}-t_{2}\right)}^{\prime}+\varphi_{o}\left(\beta_{t_{1}}-\beta_{t_{2}}\right)+0,4 \beta_{\left(t_{1}-t_{2}\right)}^{\prime}\right] \\
& \frac{\sigma}{\mathrm{E}_{\mathrm{c}}}=\left[\varphi_{o}\left(\beta_{t_{1}}-\beta_{t_{1}}\right)+0,4 \beta^{\prime}{ }_{\left(t_{2}-t_{1}\right)}\right] \frac{\sigma}{\mathrm{E}_{\mathrm{c}}}
\end{aligned}
$$


Igualando esta expresión a [2] vemos que los términos correspondientes a la deformación plástica diferida son iguales en ambas y que $\beta_{\left(t_{2}-t_{1}\right)}^{\prime}$ tendría que ser igual a $\beta_{\left(t_{3}-t_{1}\right)}^{\prime}$ caso que en realidad es imposible, pues siempre $\beta_{\left(t_{3}-t_{1}\right)}^{\prime}>\beta_{\left(t_{2}-t_{1}\right)}$.

Por consiguiente las fórmulas de deformaciones por fluencia de nuestras instrucciones EH-73 y EP-77 no admiten, como sucedía con las Recomendaciones del CEB-FIP de 1970 o con la EH-68, el principio de superposición, siendo éste un grave inconveniente a la hora de proyectar teniendo en cuenta acciones que entran en juego en distintos tiempos.

Según hemos visto al comparar [2] con [3] el problema se centra únicamente en el sumando correspondiente a la deformación elástica diferida y en consecuencia nos limitaremos a estudiar aisladamente dicho sumando.

Veamos, en primer lugar, la evolución de la deformación elástica diferida, $\varepsilon_{e d}$, al cargar en $t_{1} y$ descargar totalmente en $t_{2}$. Como 0,4 es siempre un factor constante prescindiremos de él limitándonos a estudiar $\beta^{\prime}$.

Con carga desde $t_{1}$ a $t_{2}$ obtenemos $\beta^{\prime}{ }_{\left(t_{2}-t_{1}\right)}$. Aunque nuestras instrucciones no lo expresan explícitamente, parece deducirse de ellas que al descargar en $t_{2}$ se obtendría para $t=\infty$ un valor 1 para $\beta^{\prime}$, lo que no resulta lógico dado que la deformación elástica diferida que se ha almacenado al cargar era $\beta_{\left(r_{2}-r_{1}\right)}^{\prime}<1$. Llevando el caso al límite tendríamos que cuando $t_{2}$ está infinitamente próximo a $\mathrm{t}_{1}$ (carga-descarga instantánea) la deformación elástica diferida que se ha adquirido es $\beta^{\prime}{ }_{\left(t_{2}-t_{1}\right)}=0$ y en cambio al descargar seguiremos teniendo $\beta^{\prime}=1$ para $t=\infty$.

Por consiguiente, siguiendo a Rüsch, parece conveniente limitar la recuperación elástica diferida al valor de $\beta^{\prime}{ }_{\left(t_{2}-t_{1}\right)}$ con lo que la curva de descarga será asintótica con el eje ot. (Fig. 2).

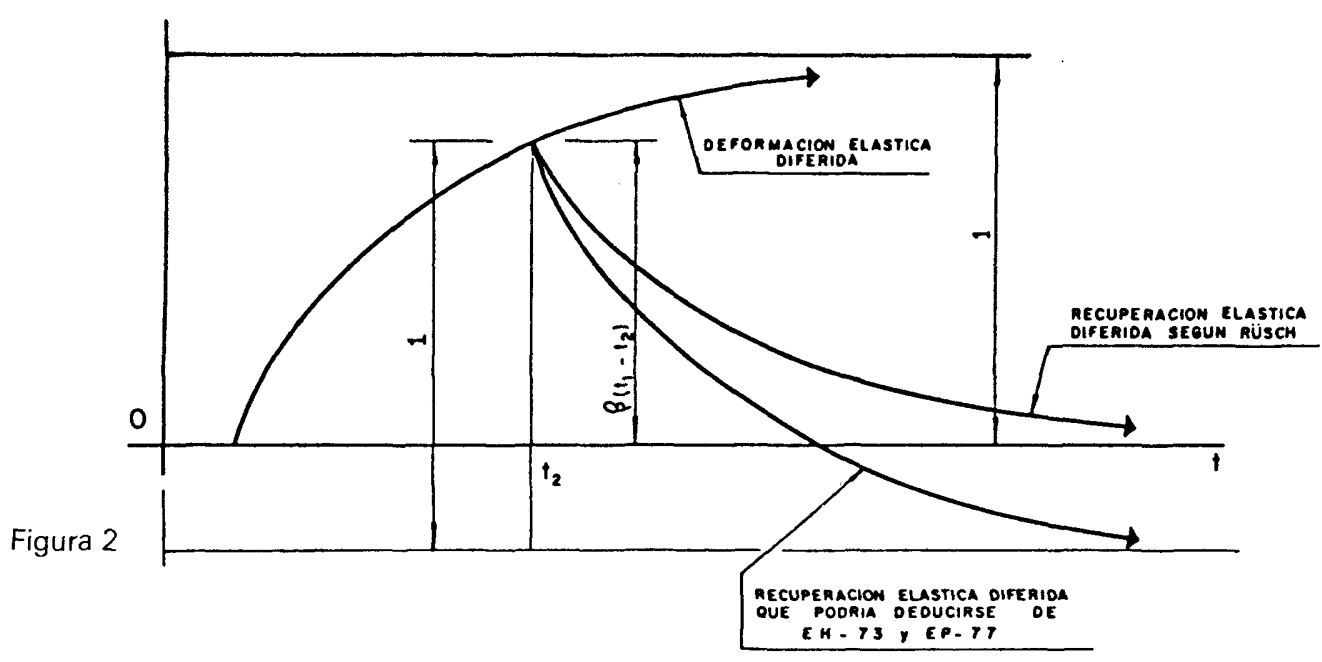

Esta consideración se recoge en la fórmula de $\varphi_{t}$ mediante la adición de un nuevo término:

$\varphi_{1}=\varphi_{o}\left(\beta_{t_{2}}-\beta_{t_{1}}\right)+0,4 \beta^{\prime}{ }_{\left(t_{2}-t_{1}\right)}-0,4 \beta^{\prime}{ }_{\left(t_{2}-t_{1}\right)} \cdot \beta^{\prime}{ }_{\left(r-t_{2}\right)}$

siendo $t$ el tiempo transcurrido desde el origen hasta el instante en que queremos hallar $\varphi_{1}$. Se supone que $t>t_{2}$. Si $t \leqslant t_{2}$ no se ha verificado aún la descarga y $\beta^{\prime}\left(1-t_{2}\right)=0$ coincidiendo la fórmula con la de nuestras instrucciones. Cuando $\mathrm{t}=\infty ; \beta^{\prime}{ }_{(1-t,)}=1 \mathrm{y}$ $\varphi_{t}=\varphi_{o}\left(\beta_{t_{2}}-\beta_{t_{1}}\right)$ lo que indica que se ha recuperado toda la deformación elástica diferida, quedando únicamente la plástica diferida.

Volveremos a nuestro caso del principio $y$ haremos las mismas operaciones de descarga y carga instantáneas en el instante $t_{2}$ pero limitándonos ahora, tal $y$ como anteriormente dijimos, al estudio de las deformaciones elásticas diferidas. 
La descarga en $t_{2}$ nos da la curva $\beta^{\prime \prime}$ (fig. 3) cuya ecuación para $t \geqslant t_{2}$ es:

$\beta^{\prime \prime}=\beta_{\left(t_{2}-t_{1}\right)}^{\prime}-\beta_{\left(t_{2}-t_{1}\right)}^{\prime} \cdot \beta^{\prime}{ }_{(t-2)}$.

La carga en $t_{2}$ nos da la curva $\beta^{\prime \prime \prime}$ que, en definitiva, es la $\beta^{\prime}$ trasladada según ot la magnitud $t_{2}-t_{1}$. En realidad, sumando $\beta^{\prime \prime}$ y $\beta^{\prime \prime \prime}$ deberíamos obtener $\beta^{\prime}$.

$\beta^{\prime \prime \prime}=\beta_{\left(t-t_{2}\right)}^{\prime}$ sumando esta expresión con la de $\beta^{\prime \prime}$ :

$\beta^{\prime \prime}+\beta^{\prime \prime \prime}=\beta^{\prime}{ }_{\left(t_{2}-t_{1}\right)}-\beta^{\prime}{ }_{\left(t_{2}-t_{1}\right)} \cdot \beta^{\prime}{ }_{\left(t-t_{2}\right)}+\beta^{\prime}{ }_{\left(t-t_{2}\right)}=\beta^{\prime}{ }_{\left(t-t_{2}\right)}\left(1-\beta_{\left(t_{2}-t_{1}\right)}^{\prime}\right)+\beta_{\left(t_{2}-t_{1}\right)}^{\prime}$.

Particularizando para $t_{3}$ :

$\beta^{\prime \prime}+\beta^{\prime \prime \prime}=\beta_{\left(t_{3}-t_{2}\right)}^{\prime}\left[1-\beta_{\left(t_{2}-t_{1}\right)}^{\prime}\right]+\beta_{\left(t_{2}-t_{1}\right)}^{\prime}$.

Expresión que, en general, no será igual a la de $\beta^{\prime}{ }_{\left(t_{3}-t_{1}\right)}$. Solamente para $t_{3}=t_{2}$ y $t_{3}=\infty$ se verifica que $\beta^{\prime}=\beta^{\prime \prime}+\beta^{\prime \prime \prime}=\beta_{\left(t_{2}-t_{1}\right)}$ y $\beta^{\prime}=\beta^{\prime \prime}+\beta^{\prime \prime \prime}=1$, respectivamente.

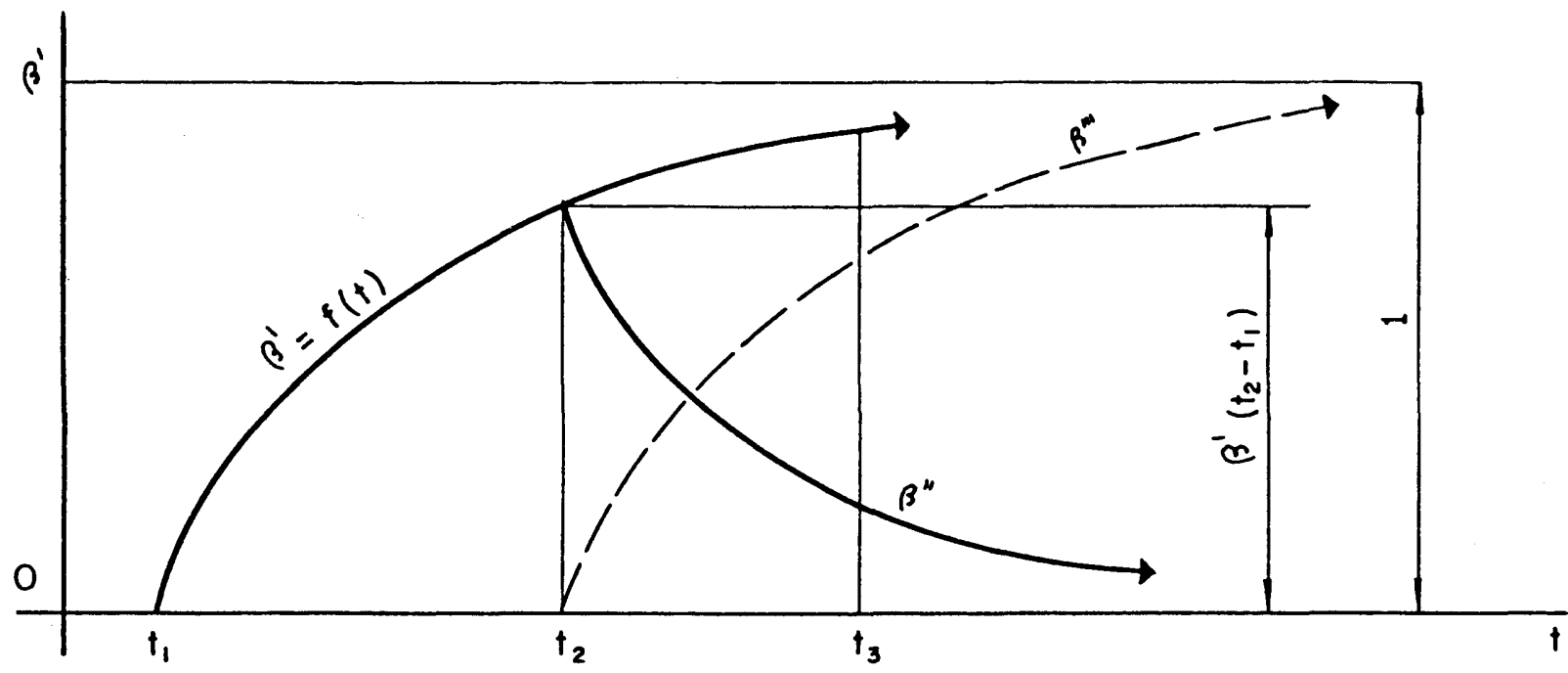

Figura 3

Por consiguiente y aun teniendo en cuenta el tercer término corrector de la recuperación elástica diferida, seguimos sin poder aplicar el principio de superposición.

Para solventar el problema partiremos de que tanto la curva $\beta^{\prime}$ como la $\beta^{\prime \prime}$ son experimentalmente ciertas. En tal caso $\beta^{\prime \prime \prime}$ se obtendrá como diferencia entre $\beta^{\prime}$ y $\beta^{\prime \prime}$ con lo que evidentemente $\beta^{\prime}=\beta^{\prime \prime}+\beta^{\prime \prime \prime}$.

$\mathrm{Si}$ en lugar de efectuar en $\mathrm{t}_{2}$ una descarga completa nos hubiéramos limitado a disminuir la tensión $\sigma$ en $\Delta \sigma$ siendo $\Delta \sigma<\sigma$, la nueva curva de deformación elástica diferida estaría comprendida entre $\beta^{\prime}$ y $\beta^{\prime \prime}$, pareciendo lógico admitir como curva de deformación la resultante de efectuar una interpolación lineal entre ambas.

Como a partir de ahora empezaremos a tener en cuenta distintos cambios de tensiones introducidos en diferentes tiempos parece conceptualmente más claro actuar con deformaciones elásticas diferidas reales $\left(\varepsilon_{e d}\right)$ que con los valores de $\beta^{\prime}$. 
Llamemos $\varepsilon_{i d}$ a la nueva curva:

$$
\varepsilon_{e d}=\varepsilon_{e d}^{\prime}-\left(\varepsilon_{e d}^{\prime}-\varepsilon^{\prime \prime}{ }_{e d}\right) \frac{\Delta \sigma}{\sigma}
$$

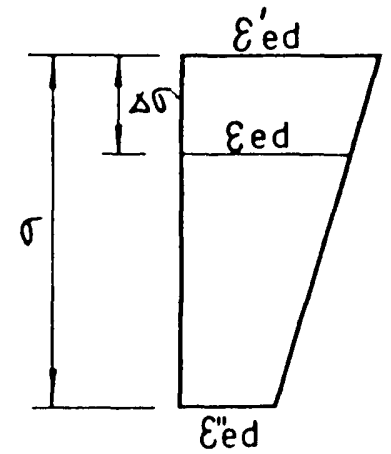

Figura 4

Particularizando para carga total durante el periodo $t_{2}-t_{1}$ y descarga parcial en $t_{3}$ :

$$
\varepsilon_{e d}=\varepsilon_{e d}^{\prime}\left(r_{3}-r_{1}\right)-\left[\varepsilon_{\left(r_{3}-t_{1}\right)}^{\prime}-\varepsilon_{e d}^{\prime}\left(r_{2}-t_{1}\right) \cdot \varepsilon_{e d}^{\prime}\left(r_{3}-t_{2}\right)\right] \frac{\Delta \sigma}{\sigma} .
$$

Otra descarga parcial en $t_{3}$ nos llevaría al mismo tipo de interpolación, considerando ahora como ley superior la anteriormente obtenida para $\varepsilon_{\text {dd }}$ y como ley inferior la correspondiente a la descarga total en $t_{3}$.

La fig. 5 nos da idea de cómo pueden ir obteniéndose las deformaciones o recuperaciones elásticas cuando existen sucesivos escalones de descarga parcial.

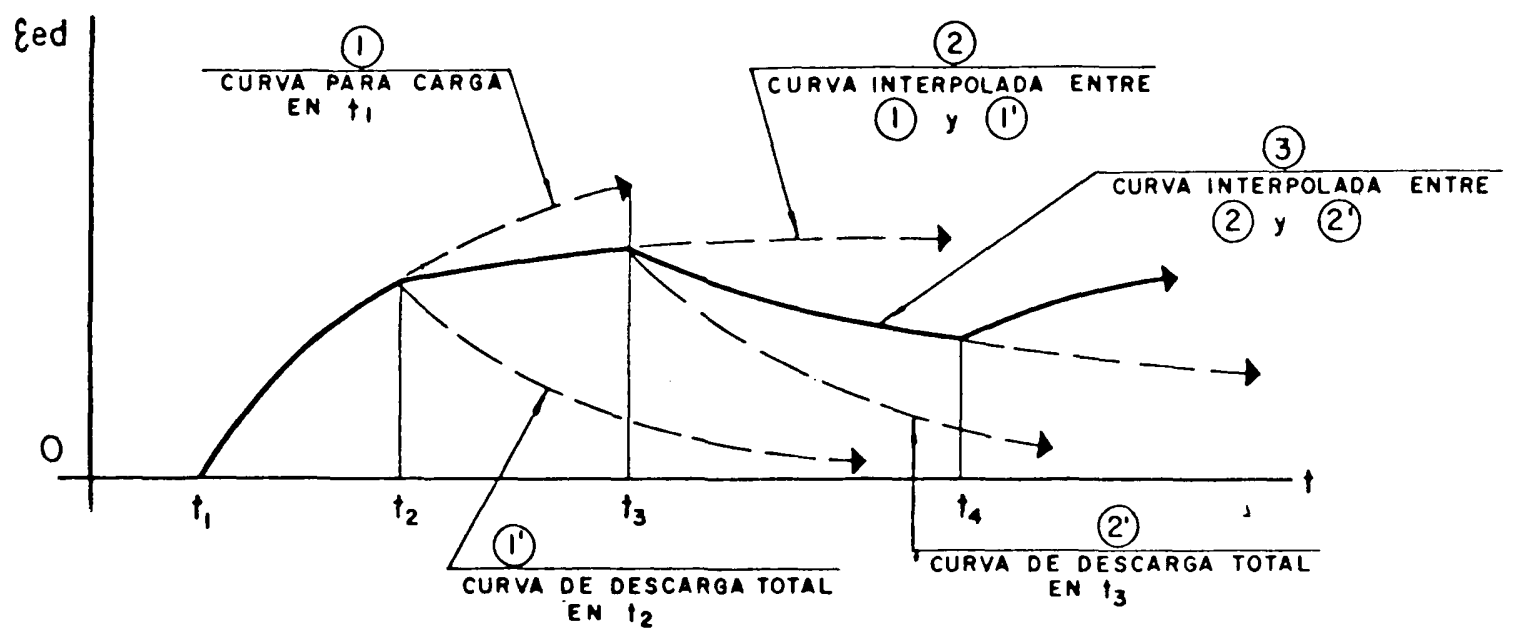

Figura 5

Si en $t_{4}$ en lugar de tener una disminución de la carga hubiera habido un aumento de ella deberíamos sumar, a la curva (3), la curva de la deformación elástica diferida que corresponde a este incremento de tensión.

Cuando han de intervenir varias acciones, con sus correspondientes cambios tensionales, el proceso indicado puede resultar engorroso, pero es la única forma de hacer adecuadamente compatibles el concepto de deformación elástica diferida con el cálculo de deformaciones por fluencia preconizado por la EH-73 y EP-77 en caso de carga mantenida. 
Ilustraremos las conclusiones con un ejemplo:

Supongamos que a los 7 días de su hormigonado se somete una probeta de $15 \mathrm{~cm}$ de diámetro a una tensión de $50 \mathrm{~kg} / \mathrm{cm}^{2}$ y 23 días después se incrementa esta tensión en $100 \mathrm{~kg} / \mathrm{cm}^{2}$. Transcurridos 40 días más se disminuye la tensión en $40 \mathrm{~kg} / \mathrm{cm}^{2}$ y 20 dias después en $20 \mathrm{~kg} / \mathrm{cm}^{2}$.

El hormigón tiene una consistencia plástica y su módulo de elasticidad a los 28 dias de edad es de $350.000 \mathrm{~kg} / \mathrm{cm}^{2}$.

La temperatura de $20^{\circ} \mathrm{C}$ se mantiene constante a lo largo de todo el proceso pudiéndose admitir una humedad relativa media del $70 \%$.

Se desea conocer las deformaciones unitarias diferidas por fluencia a los 30,70 y 90 días así como para $\mathrm{t}=\infty$ :

$$
\begin{array}{lll}
\mathrm{t}_{1}=7 \text { dias } & \mathrm{t}_{2}=30 \text { dias } & \mathrm{t}_{3}=70 \text { dias } \quad \mathrm{t}_{4}=90 \text { dias } \quad \mathrm{t}_{5}=\infty \\
\varphi_{\rho^{\prime}}=2,0 & \alpha=1,5 & \mathrm{e}=1,5 \times \frac{2 \pi \mathrm{r}^{2}}{2 \pi \mathrm{r}}=1,5 \times 7,5=11,25 \mathrm{~cm} .
\end{array}
$$

Hallaremos, en primer lugar, las deformaciones plásticas diferidas. Con las curvas de la Instrucción:

$\begin{array}{lccccl}\text { Días: } & 7 & 30 & 70 & 90 & \infty \\ \beta_{j}^{\prime} \text { o } \beta,: & 0,24 & 0,40 & 0,52 & 0,56 & 0,94\end{array}$

Deformaciones instantáneas por las distintas cargas:

A los 7 días, carga de $50 \mathrm{~kg} / \mathrm{cm}^{2} \frac{\sigma}{E_{c}}=\frac{50}{350.000}$

A los 30 días, carga de $100 \mathrm{~kg} / \mathrm{cm}^{2} \frac{\sigma}{E_{c}}=\frac{100}{350.000}$

A los 70 días, carga de $40 \mathrm{~kg} / \mathrm{cm}^{2} \frac{\sigma}{E_{c}}=\frac{40}{350.000}$

Deformaciones plásticas diferidas:

A los 30 dias: $\frac{50}{350.000} \times 2(0,40-0,24)=4,57 \times 10^{-5}$

A los 70 dias: $\frac{50}{350.000} \times 2(0,52-0,24)+\frac{100}{350.000} \times 2(0,52-0,40)=14,86 \times 10^{-5}$ 
A los 90 dias: $\frac{50}{350.000} \times 2(0,56-0,24)+\frac{100}{350.000} \times 2(0,56-0,40)-\frac{40}{350.000} \times$

$\times 2(0,56-0,52)=17,83 \times 10^{-5}$

$\begin{aligned} \text { Para } t=\infty \quad & \frac{50}{350.000} \times 2(0,94-0,24)+\frac{100}{350.000} \times 2(0,94-0,40)-\frac{40}{350.000} \times \\ & \times 2(0,94-0,52)-\frac{20}{350.000} \times 2(0,94-0,56)=36,91 \times 10^{-5}\end{aligned}$

Realizaremos ahora el cálculo de las deformaciones elásticas diferidas. Es conveniente realizar a priori un esquema de evolución con el tiempo como el de la figura 6.

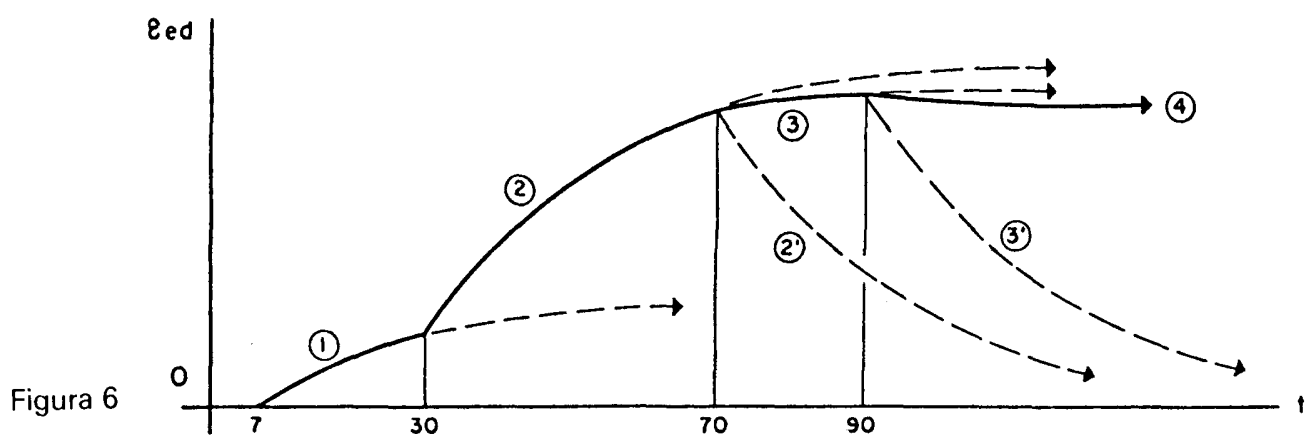

En este esquema las distintas curvas representan los valores que van tomando las deformaciones elásticas diferidas.

Curva (1) $\varepsilon_{e d}$ correspondiente a $50 \mathrm{~kg} / \mathrm{cm}^{2}$ que empiezan a actuar en $\mathrm{t}=7$ días.

Curva (2) Curva (1) más $\varepsilon_{e d}$ correspondiente a $100 \mathrm{~kg} / \mathrm{cm}^{2}$ que empiezan a actuar en $\mathrm{t}=30$ dias.

Curva (2) Descarga total de $150 \mathrm{~kg}$ en $\mathrm{t}=70$ dias.

Curva (3) Curva interpolada entre (2) y (2) para una descarga de $40 \mathrm{~kg} / \mathrm{cm}^{2}$ en t=70 días.

Curva (3) Descarga total de $110 \mathrm{~kg} / \mathrm{cm}^{2}$ en $\mathrm{t}=90$ días.

Curva (4) Curva interpolada entre la (3) y la (3).

$\mathrm{t}=30 \quad \beta_{(30-7)}^{\prime}=0,55 \quad \varepsilon_{\text {ed }}=0,4 \frac{50}{350.000} \times 0,55=3,14 \times 10^{-5}$ 


$$
\begin{aligned}
& \mathbf{t}=70\left\{\begin{array}{l}
\beta^{\prime}(70-7)=0,70 \\
\beta^{\prime}(70-30)=0,62
\end{array} \varepsilon_{e d}=0,4 \frac{50}{350.000} \times 0,70+0,4 \frac{100}{350.000} \times 0,62=11,09 \times 10^{-5}\right. \\
& t=90\left\{\begin{array}{llll}
\beta^{\prime}{ }_{(90-7)}=0,75 & \text { En curva (2): } \varepsilon_{\text {ed }}=0,4 \frac{50}{350.000} \times 0,75+0,4 \frac{100}{350.000} \times \\
\beta^{\prime}{ }_{(90-30)}=0,69 & & \times 0,69=12,17 \times 10^{-5} \\
\beta^{\prime}{ }_{(90-70)}=0,52 &
\end{array}\right.
\end{aligned}
$$

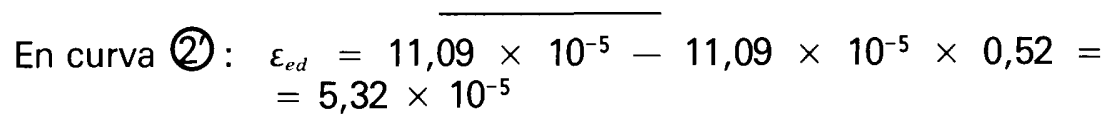

Interpolando entre (2) $y$ (2) (fig. 7) $\varepsilon_{e d}=\left(12,17-\frac{12,17-5,32}{150} 40\right) \times 10^{-5}=10,34 \times 10^{-5}$

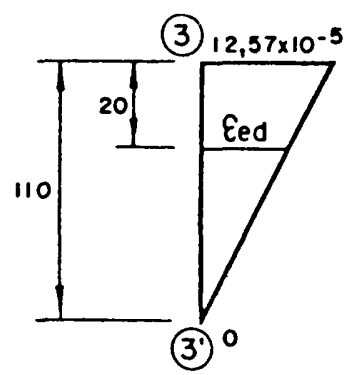

Figura 7

$t=\infty \quad \beta^{\prime}=1$

En curva (2) $\varepsilon_{e d}=0,4 \frac{50}{350.000}+0,4 \frac{100}{350.000}=17,14 \times 10^{-5}$

En curva (2) $\varepsilon_{e d}=0$

En curva (3) Interpolando entre (2) y (2) (fig. 8) $\varepsilon_{e d}=17,14$ :

$$
\varepsilon_{e d}=\left(17,14-\frac{17,14}{150} 40\right) \times 10^{-5}=12,57 \times 10^{-5}
$$

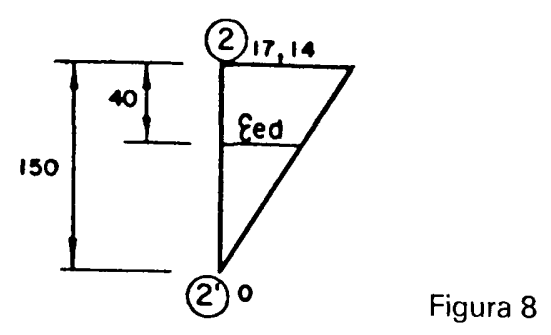


En curva (3) $\varepsilon_{c \cdot d}=0$

En curva (4) Interpolamos entre (3) y (3) (fig. 9).

$$
\left(12,57-\frac{12,57}{110} 20\right) \times 10^{-5}=10,25 \times 10^{-5}
$$

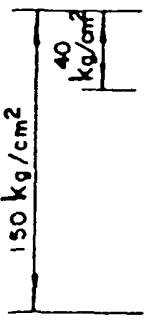

Figura 9
(2)

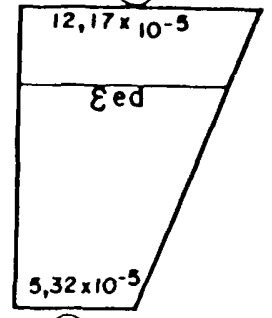

(2)

Resumiendo y sumando las deformaciones plásticas diferidas con las correspondientes elásticas diferidas tendremos el siguiente cuadro:

\begin{tabular}{|c|c|c|c|}
\hline \multirow[b]{2}{*}{1} & \multicolumn{3}{|c|}{ DEFORMACIONES POR FLUENCIA $10^{-5}$} \\
\hline & $\begin{array}{l}\text { PLASTICAS } \\
\text { OIFERIOAS }\end{array}$ & $\begin{array}{l}\text { ELASTICAS } \\
\text { DIFERIOAS }\end{array}$ & TOTALES \\
\hline 7 & 0 & 0 & 0 \\
\hline & 4,57 & 3,14 & 7,71 \\
\hline & 14,86 & 11,09 & 25,95 \\
\hline & 17,83 & 10,34 & 28,17 \\
\hline & 36,91 & 10,25 & $46,16-$ \\
\hline
\end{tabular}

\section{résumé}

\section{DEFORMATIONS DANS LE BETON PAR FLUAGE POUR UN ETAT TENSIONNEL DISCONTINU AVEC LE TEMPS}

\section{José Ramón Rosso Ródenas}

Ingénieur technique des Travaux Publics

Le principe de superposition admis par les Recommandations du CEB-FIP de 1970 et pa celles du code EH-68 n'est pas valable pour les formules de déformations par fluage des codes actuels EH-73 et EP-77, ce qui constitue un grave inconvénient pour calculer une structure lorsque des charges agissent d'une manière discontinue avec le temps.

Cet article a pour but d'établir une méthode permettan de calculer une courbe de fluage pour une décharge intermédiaire qui observe le principe de superposition, admettant comme certaines la courbe de fluage et la courbe de déformation élastique différée pour une décharge totale des codes EH-73 et EP-77.

\section{summary}

DEFORMATIONS IN CONCRETE DUE TO CREEP FOR A DISCONTINUOUS TENSIONAL STATE IN TIME

\section{José Ramón Rosso Ródenas}

Public Works Technical Engineer

The overlap principle admitted in the CEB-FIP 1970 Recommendations and in the EH-68 is not valid for deformation formulae due to EP.77 and it represents a serious setback when designing a structure when loads are acting that have a discontinuous way in time.

The purpose of this article is to arbitrate a method that will enable us to calculate a creep curve for an intermediary discharge that creep curve for an intermediary discharge that fulfils the overlap principle, admitting the load creep curve and the deferred elastic defor-
mation one for total compliance with the mation one for
EH-73 and EP. 77.

\section{zusammenfassung}

BETONFLIESSVERFORMUNGEN BEI EINEM ZEITLICH NICHT KONTINUIER LICHEM SPANNUNGSZUSTAND

\section{José Ramón Rosso Ródenas}

Technischer Ingenieur für Oeffentliche Bauten

Der in den Empfehlungen des CEB-FIP von 1970 und in der EH-68 angenommene Grund satz der Ueberlagerung gilt nicht für die Fliessverformungsformeln der gegenwärtigen Anweisungen EH-73 und EP-77, was im Falle von zeitlich nicht kontinuierlichen $\mathrm{Be}$ lastungen einen grossen Nachteil für die Projektierung von Strukturen darstellt.

Zweck dieses Artikels ist es, eine Methode fü die Berechnung einer Fliesskurve für eine mittlere Entlastung festzulegen, welche dem Grundsatz der Ueberlagerung Rechnung trägt, wobei die Lastfliesskurve und diejenige de verzögerten Verformung für die Gesamten tlastung der EH-73 und EP-77 als gültig ange nommen werden. 